\title{
Overall Postoperative Morbidity and Pancreatic Fistula Are Relatively Higher after Central Pancreatectomy than Distal Pancreatic Resection: A Systematic Review and Meta-Analysis
}

\author{
Parbatraj Regmi, ${ }^{1}$ Qing Yang, ${ }^{2}$ Hai-Jie Hu, ${ }^{1}$ Fei Liu, ${ }^{1}$ Hare Ram Karn, ${ }^{1}$ Wen-Jie Ma, ${ }^{1}$ \\ Cong-Dun Ran, ${ }^{1}$ and Fu-Yu Li (D) ${ }^{1}$ \\ ${ }^{1}$ Department of Biliary Surgery, West China Hospital of Sichuan University, Chengdu 610041, Sichuan Province, China \\ ${ }^{2}$ Department of General Surgery, The Third People's Hospital of Chengdu, \\ The Affiliated Hospital of Southwest Jiaotong University, \\ The Second Medical School of Chengdu Affiliated to Chongqing Medical University, Chengdu, Sichuan 610031, China
}

Correspondence should be addressed to Fu-Yu Li; lfy_74@hotmail.com

Received 17 September 2019; Revised 28 January 2020; Accepted 29 January 2020; Published 22 February 2020

Academic Editor: Raffaele Serra

Copyright (c) 2020 Parbatraj Regmi et al. This is an open access article distributed under the Creative Commons Attribution License, which permits unrestricted use, distribution, and reproduction in any medium, provided the original work is properly cited.

\begin{abstract}
Objective. To compare the intraoperative and postoperative outcomes of central pancreatectomy (CP) with distal pancreatectomy (DP). Methods. A systematic literature search was performed on electronic databases from MEDLINE, Embase, and PubMed from 1998 to 2018. Statistical analysis and meta-analysis were performed using statistics/data analysis (Stata ${ }^{\circledR}$ ) software, version 12.0 (StataCorp LP, College Station, Texas 77845, USA). Dichotomous variables were analyzed by estimation of relative risk (RR) with a 95 percent (\%) confidence interval (CI) and continuous variables were analyzed by standardized mean differences (SMD) with 95\% CI. Results. Twenty-four studies with 593 CP and 1226 DP were included in the meta-analysis. CP had significantly longer operation time (SMD: $1.03 ; 95 \%$ CI 0.62 to $1.44 ; P<0.001$ ) and lengthier postoperative hospital stay (SMD: $0.63 ; 95 \%$ CI 0.20 to $1.05 ; P<0.01$ ). Estimated blood loss was significantly lower in CP (SMD: $-0.34 ; 95 \%$ CI -0.58 to $-0.09 ; P=0.007$ ). Overall postoperative morbidity (RR: 1.30; $95 \%$ CI: 1.13 to 1.50 ; $P<0.001$ ), overall pancreatic fistula (RR: $1.41 ; 95 \%$ CI: 1.20 to 1.66 ; $P<0.001$ ), clinically relevant fistula (RR: $1.64 ; 95 \%$ CI: 1.25 to $2.16 ; P<0.001$ ), and postoperative hemorrhage (RR: $1.90 ; 95 \%$ CI: 1.18 to 3.06; $P<0.05)$ were all significantly higher after $\mathrm{CP}$. On long-term follow-up, DP patients were more likely to have postoperative exocrine (RR: 0.56 ; $95 \%$ CI: 0.37 to $0.84 ; P<0.05$ ) and endocrine (RR: $0.27 ; 95 \%$ CI: 0.18 to $0.40 ; P<0.001$ ) insufficiency. There was no statistically significant difference in transfusion requirement, postoperative mortality, reoperation, and tumor recurrence. Conclusion. CP is associated with significantly higher morbidity and clinically relevant pancreatic fistula. CP should only be reserved for selected patients who require postoperative pancreatic function preservation.
\end{abstract}

\section{Introduction}

Complete surgical resection is the only potentially curative treatment for pancreatic cancer. However, only $15-20 \%$ of patients are amenable to resection on initial diagnosis [1-3]. The distal pancreatectomy (DP) is considered as a standard surgical procedure for lesions located in the pancreatic neck and body [2]. Unfortunately, during the resection of benign and low-malignant lesions, normal pancreatic parenchyma is resected in the DP and may result in loss of pancreatic function and possible postoperative exocrine and endocrine impairment. After the introduction of the first central pancreatectomy (CP) with reconstruction by Dagradi and Serio in 1982, this procedure has been used as a parenchyma-preserving surgical procedure for resection of benign and low-malignant lesions of neck and proximal body of pancreas. After that, the procedure has been advanced gradually from open surgery to laparoscopic and robotic approaches $[4,5]$. 


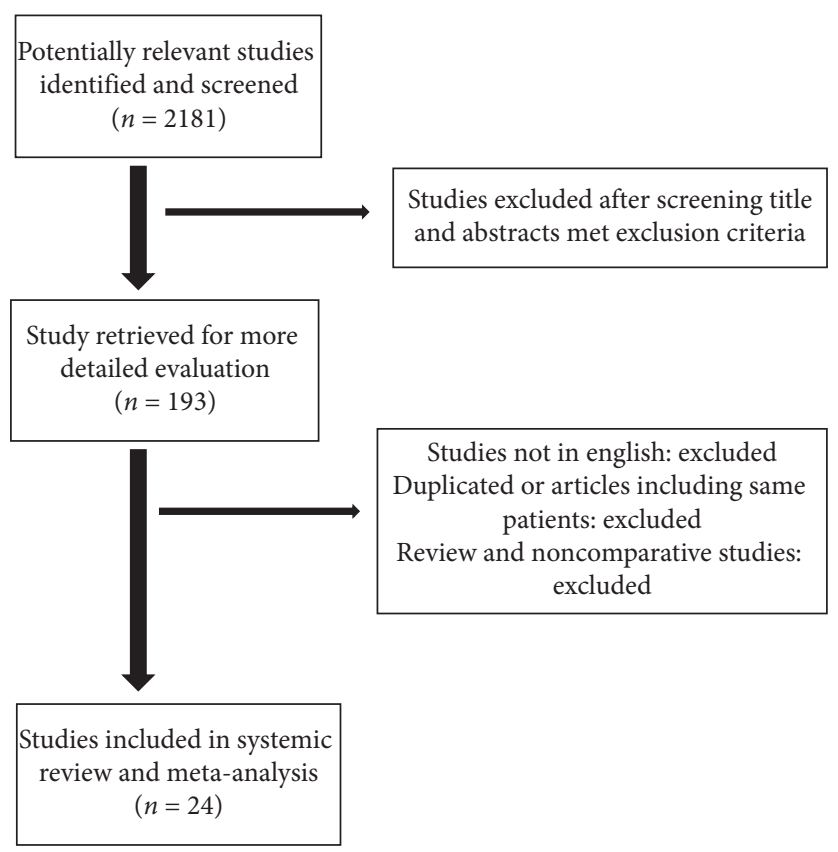

Figure 1: Prisma flow chart.

TABLE 1: Baseline characteristics of included studies.

\begin{tabular}{lcccccc}
\hline Authors & Country & Year & Group (CP/DP) & Gender (M/F) & Approach & Study type \\
\hline Yamaguchi et al. [17] & Japan & 2000 & $10 / 47$ & $27 / 30$ & Open & Retrospective \\
Balzano et al. [29] & Italy & 2003 & $32 / 21$ & $16 / 37$ & Open & Retrospective \\
Su et al. [31] & Taiwan & 2004 & $5 / 11$ & - & Open & Retrospective \\
Shibata et al. [15] & Japan & 2004 & $10 / 7$ & $6 / 11$ & Open & Retrospective \\
Müller et al. [28] & Germany & 2006 & $40 / 40$ & $38 / 42$ & Open & Prospective \\
Crippa et al. [36] & Italy and USA & 2007 & $100 / 45$ & $37 / 108$ & Open & Retrospective \\
Ocuin et al. [22] & USA & 2008 & $13 / 18$ & $8 / 23$ & Open & Retrospective \\
Hirono et al. [18] & Japan & 2009 & $24 / 28$ & $19 / 33$ & Open & Retrospective \\
Cataldegirmen et al. [27] & Germany & 2010 & $35 / 35$ & $34 / 36$ & Open & Retrospective \\
DiNorcia et al. [7] & USA & 2010 & $50 / 50$ & $26 / 74$ & Open & Retrospective \\
Lee et al. [25] & Korea & 2010 & $14 / 143$ & $56 / 101$ & Open & Retrospective \\
Shikano et al. [16] & Japan & 2010 & $26 / 35$ & $30 / 31$ & Open & Retrospective \\
Kang et al. [24] & Korea & 2011 & $17 / 22$ & $15 / 24$ & Open & Retrospective \\
Dumitrascu et al. [34] & Romania & 2012 & $22 / 25$ & $11 / 36$ & Open & Retrospective \\
Xiang et al. [20] & China & 2012 & $44 / 45$ & $36 / 63$ & Open & Retrospective \\
Du et al. [19] & China & 2013 & $36 / 26$ & $20 / 42$ & Open & Retrospective \\
Zhan et al. [21] & China & 2013 & $10 / 16$ & - & Robotic & Retrospective \\
Zureikat et al. [23] & USA & 2013 & $13 / 83$ & - & Robotic & Retrospective \\
Song et al. [26] & Korea & 2014 & $26 / 96$ & $34 / 88$ & Laparoscopic & Retrospective \\
Mise et al. [14] & Japan & 2014 & $8 / 8$ & $12 / 4$ & Open & Retrospective \\
Herrera-Cabezón et al. [32] & Spain & 2015 & $10 / 105$ & $57 / 58$ & Open & Prospective \\
Jilesen et al. [33] & Netherlands & 2015 & $8 / 72$ & $30 / 50$ & Open & Retrospective \\
Dokmak et al. [35] & France & 2017 & $35 / 165$ & $74 / 126$ & Laparoscopic & Retrospective \\
Boggi et al. [30] & Italy & 2016 & $5 / 83$ & - & Robotic & Retrospective
\end{tabular}

TABle 2: Results of meta-analysis: CP versus DP.

\begin{tabular}{lccccccc}
\hline Outcome of interest & Studies no. & Patients no. (CP/DP) & RR or SMD & 95\% CI & $P$ value & $I^{2}(\%)$ & Model \\
\hline Intraoperative outcomes & & & & & & & \\
Operation time (min) & 20 & $526 / 911$ & 1.03 & $(0.62,1.44)$ & $<0.001$ & $90 \%$ & Random effects \\
EBL (ml) & 18 & $478 / 793$ & -0.34 & $(-0.58,-0.09)$ & 0.007 & $70.6 \%$ & Random effects \\
Transfusion $(n)$ & 13 & $361 / 623$ & 0.69 & $(0.47,1.01)$ & 0.059 & 0.0 & Fixed effects \\
\hline
\end{tabular}


TABLE 2: Continued.

\begin{tabular}{lccccccc}
\hline Outcome of interest & Studies no. & Patients no. (CP/DP) & RR or SMD & $95 \%$ CI & $P$ value & $I^{2}(\%)$ & Model \\
\hline Postoperative outcomes & & & & & & & \\
$\quad$ & & & & & & \\
LOS (days) & 23 & $499 / 1002$ & 1.30 & $(1.13,1.50)$ & $<0.001$ & 0.0 & Fixed effects \\
Overall complications & 21 & $593 / 1226$ & 1.41 & $(1.20,1.66)$ & $<0.001$ & 0.0 & Fixed effects \\
Overall PF & 24 & $350 / 825$ & 1.64 & $(1.25,2.16)$ & $<0.001$ & 1.7 & Fixed effects \\
PF (grade B + C) & 12 & $373 / 567$ & 1.90 & $(1.18,3.06)$ & 0.008 & 0.0 & Fixed effects \\
POH & 11 & $411 / 805$ & 1.10 & $(0.69,1.73)$ & 0.699 & 6.1 & Fixed effects \\
Reoperation ( $n$ ) & 15 & $63 / 163$ & 3.31 & $(0.52,21.32)$ & 0.207 & 0.0 & Fixed effects \\
$\quad$ Perioperative mortality & 3 & & & & & \\
Long-term outcomes & & $483 / 694$ & 0.27 & $(0.18,0.40)$ & $<0.001$ & 0.0 & Fixed effects \\
Endocrine impairment & 18 & $227 / 163$ & 0.15 & $(0.06,0.42)$ & $<0.001$ & 0.0 & Fixed effects \\
IDDM & 6 & $304 / 324$ & 0.56 & $(0.37,0.84)$ & 0.006 & 0.0 & Fixed effects \\
Exocrine impairment & 9 & $155 / 366$ & 1.02 & $(0.48,2.20)$ & 0.956 & 0.0 & Fixed effects \\
Recurrence & 7 & & &
\end{tabular}

EBL: estimated blood loss; LOS: length of hospital stay; PF: pancreatic fistula; POH: postoperative hemorrhage; IDDM: insulin-dependent diabetes mellitus.

TABLE 3: Surgical pathology of included studies.

\begin{tabular}{|c|c|c|}
\hline \multirow{2}{*}{ Authors } & \multicolumn{2}{|c|}{ Surgical pathology } \\
\hline & Benign or borderline (CP/DP) & Malignant (CP/DP) \\
\hline Yamaguchi et al. [17] & $10 / 47$ & $0 / 0$ \\
\hline Balzano et al. [29] & $32 / 21$ & $0 / 0$ \\
\hline Su et al. [31] & $5 / 11$ & $0 / 0$ \\
\hline \multicolumn{3}{|l|}{ Shibata et al. [15] } \\
\hline Müller et al. [28] & $36 / 36$ & $4 / 4$ \\
\hline Crippa et al. [36] & $93 / 17$ & $7 / 28$ \\
\hline Ocuin et al. [22] & $13 / 18$ & $0 / 0$ \\
\hline Hirono et al. [18] & $20 / 24$ & $4 / 4$ \\
\hline Cataldegirman et al. [27] & $32 / 34$ & $3 / 1$ \\
\hline DiNorcia et al. [7] & $50 / 46$ & $0 / 4$ \\
\hline Lee et al. [25] & $14 / 121$ & $0 / 22$ \\
\hline Shikano et al. [16] & $24 / 33$ & $2 / 2$ \\
\hline Kang et al. [24] & $17 / 22$ & $0 / 0$ \\
\hline Dumitrascu et al. [34] & $19 / 21$ & $3 / 4$ \\
\hline Xiang et al. [20] & $44 / 55$ & $0 / 0$ \\
\hline Du et al. [19] & $36 / 26$ & $0 / 0$ \\
\hline Zhan et al. [21] & $10 / 12$ & $0 / 4$ \\
\hline Zureikat et al. [23] & $13 / 6$ & $0 / 77$ \\
\hline Song et al. [26] & $26 / 96$ & $0 / 0$ \\
\hline Mise et al. [14] & $8 / 8$ & $0 / 0$ \\
\hline Herrera-Cabezón et al. [32] & $10 / 82$ & $0 / 23$ \\
\hline Jilesen et al. [33] & $8 / 51$ & $0 / 21$ \\
\hline Dokmak et al. [35] & $25 / 102$ & $10 / 63$ \\
\hline Boggi et al. [30] & & \\
\hline
\end{tabular}

CP: central pancreatectomy; DP: distal pancreatectomy.

Current literature has reported a relatively higher incidence of postoperative new-onset diabetes mellitus after DP than CP and pancreaticoduodenectomy (PD) $[6,7]$. Kang et al. have reported that resected pancreatic volume was an independent risk factor for postoperative endocrine impairment [8]. In CP, the volume of remnant pancreas is responsible for maintaining postoperative endocrine and exocrine function, but there are still controversies regarding the management of additional pancreatic stump. The morbidity following CP is comparatively higher than other standard pancreatic resections, pancreatic fistula being major morbidity
$[9,10]$. CP has relative benefits of preserving normal pancreatic parenchyma and spleen, but potential challenges to reconstruct additional pancreatic stump and high incidence of postoperative pancreatic fistula create a dilemma to choose appropriate surgical procedure.

Expected benefits and potential complications create controversies in selecting surgical procedures for lesions of the pancreatic neck and proximal body, so this study was aimed to review all the relevant electronic databases to evaluate and compare intraoperative, short- and long-term, and postoperative outcomes following CP and DP. 


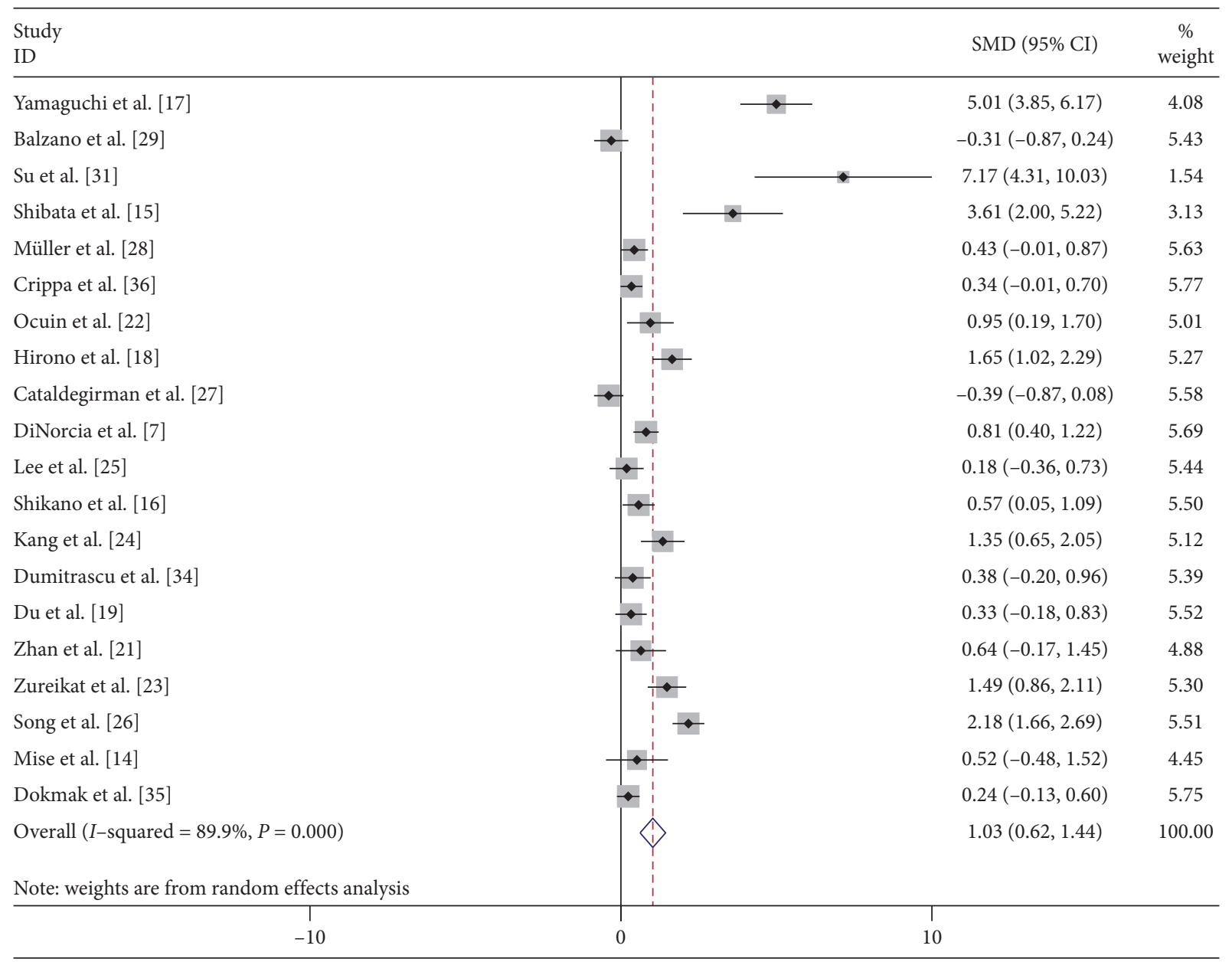

FIGURE 2: Forest plot comparing duration of operation for central versus distal pancreatectomy.

\section{Methods}

The systematic review and meta-analysis were performed according to the Preferred Reporting Items for the Systematic Review and Meta-Analysis Protocols (PRISMA-P) guideline [11].

2.1. Search Strategy. A systematic literature search was performed on electronic databases from Ovid MEDLINE (R), Embase, and PubMed from 1 January 1998 to 31 December 2017. Search headings used were "(central pancreatectomy OR medial pancreatectomy OR middle pancreatectomy OR segmental pancreatectomy) and (distal pancreatectomy OR left pancreatectomy)". Searches were performed without any restrictions and all the abstracts, studies, and citations were reviewed. 2181 studies were found after the comprehensive search of the database. 1988 articles were excluded after screening the title abstracts and duplicated materials. 193 studies were evaluated in detail and ultimately 24 eligible studies were included in systematic review and meta-analysis (Figure 1).
2.2. Study Selection and Quality Assessment. Two reviewers independently screened all the selected citations independently. Any disagreement between the two reviewers was resolved by discussion with the corresponding author. All the retrospective and prospective matched pairs and nonmatched pairs comparing CP and DP were extracted.

Inclusion criteria were

(1) Original English articles;

(2) Patients with benign pathology of low-malignant tumors of the pancreatic neck or proximal body;

(3) Studies comparing the clinical outcomes between CP and DP;

(4) Studies that provided adequate information about demographic characteristics and intraoperative and postoperative outcomes.

Exclusion criteria were

(1) Original articles with <5 CP or DP patients;

(2) Abstracts, expert opinion, reviews, editorials, and letter to the editor; 


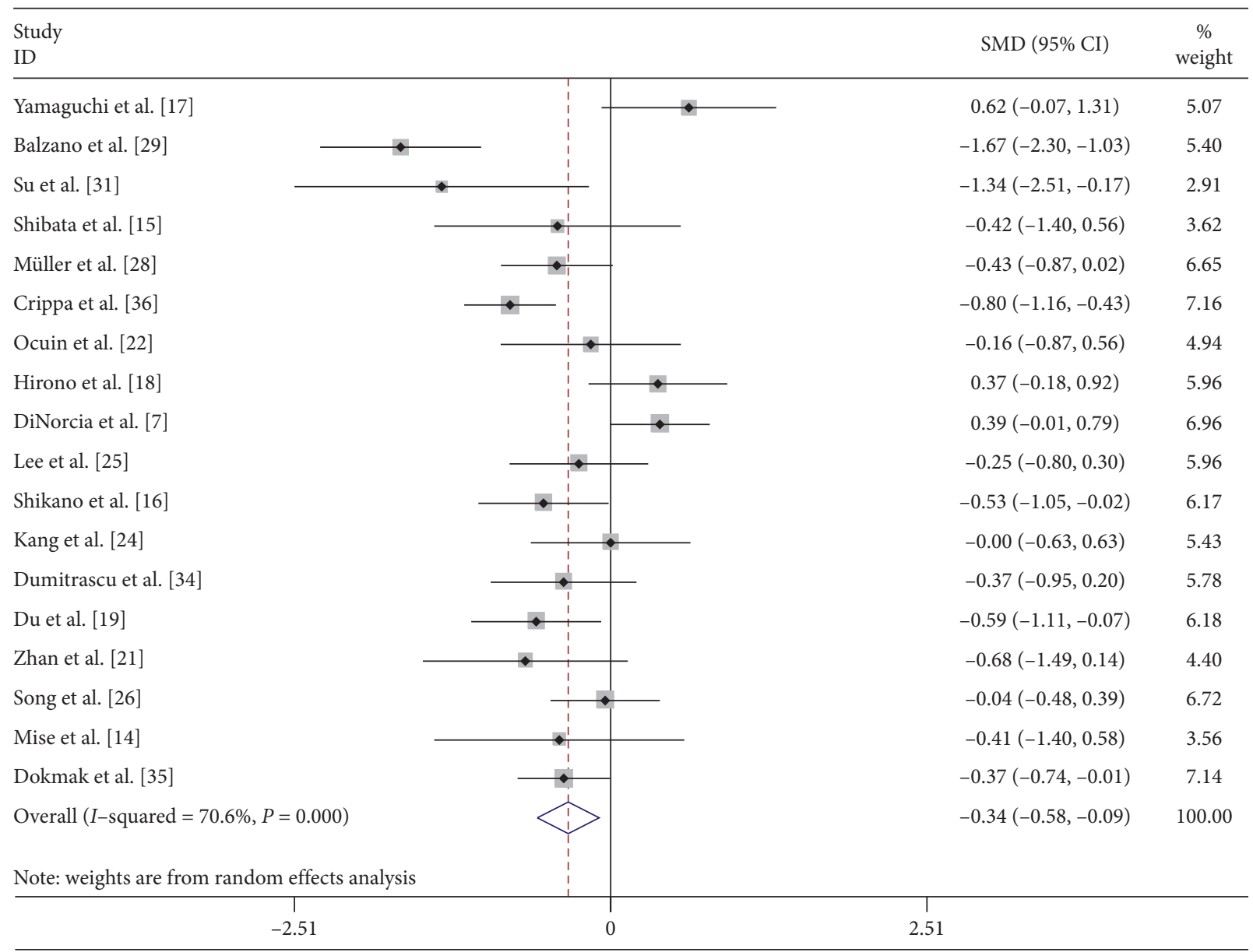

FIGURE 3: Forest plot comparing estimated blood loss for central versus distal pancreatectomy.

(3) Studies that lack adequate clinical data on intraoperative and postoperative outcomes.

Quality assessment was done by the Newcastle Ottawa Scale (NOS). Studies selected had a score above 5. Scoring criteria were based on the selection of study groups, the comparability of groups, and the ascertainment of either exposure or outcome. One study had 9, 3 had 8, 17 had 7, and 3 had 6 out of possible 9 scores.

2.3. Data Extraction. Data were extracted for (a) demographic characteristics, (b) intraoperative outcomes (operation time, intraoperative blood loss, and transfusion requirement), (b) short-term postoperative outcomes (postoperative hospital stay, overall morbidity, pancreatic fistula, clinically relevant pancreatic fistula, postoperative hemorrhage, reoperation, and 30-day mortality), and (c) long-term outcomes (overall endocrine function, Insulindependent diabetes mellitus, exocrine function, and tumor recurrence). If two articles were published by the same authors or from the same institution, a comparatively more informative study with the maximum population was selected. When data was found in median and range, the mean and standard deviation was estimated as described by Wan et al. and Luo et al. [12, 13].
2.4. Statistical Analysis. Statistical analysis and meta-analysis were performed by statistics/data analysis (Stata ${ }^{\circledR}$ ) software, version 12.0 (StataCorp LP, College Station, Texas 77845 USA). Dichotomous variables were analyzed by estimation of relative risk (RR) with a 95 percent (\%) confidence interval (CI) and continuous variables were analyzed by standardized mean differences (SMD)/weighted mean differences (WMD) with $95 \%$ CI. $P$ value $<0.05$ was considered a statistically significant difference between the two groups. Heterogeneity was defined as low, moderate, and high based on I square value ( $<25 \%$ : low; $25-75 \%$ : moderate; $>75 \%$ : high). Heterogeneity with a high $I$ square value $>30 \%$ and $P$ value $<0.05$ was considered statistically significant. Fixed effect (Mantel-Haenszel) model was used when there was no significant heterogeneity and the random effects (DerSimonian and Laird) model was used for those with significant heterogeneity. Publication bias was examined in a funnel plot using Begg's and Egger's tests. Publication bias was considered to be present when the $P$ value was $<0.1$.

\section{Results}

3.1. Study Selection and Characteristics. Twenty-four studies performed from 1998 to 2018 were included in metaanalysis following a comprehensive literature search 


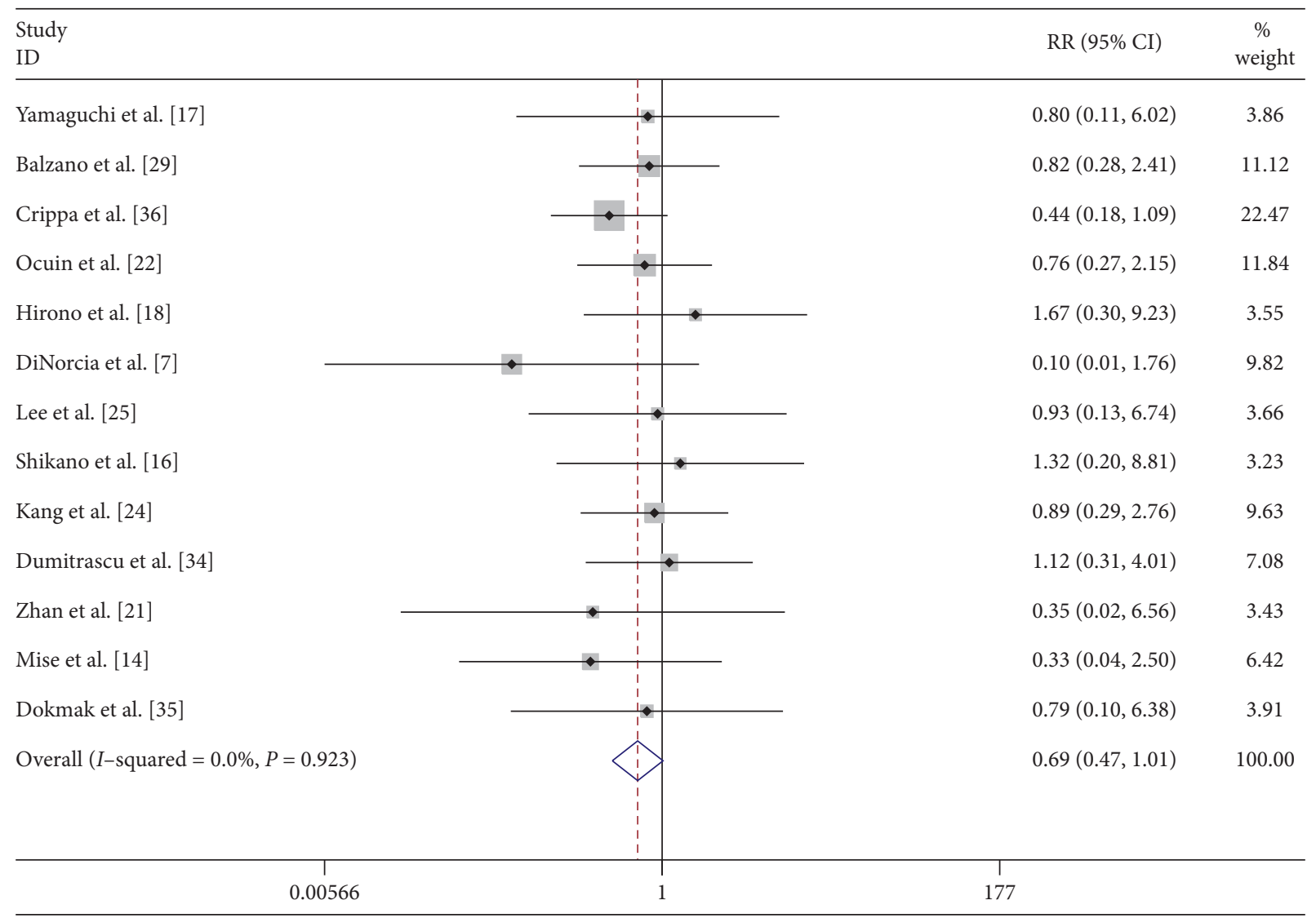

FIGURE 4: Forest plot comparing transfusion requirement for central versus distal pancreatectomy.

(Table 1). Central pancreatectomy and distal pancreatectomy were performed in 593 and 1226 patients, respectively. Of these 24 studies, five studies were performed in Japan [14-18], three each in China [19-21], USA $[7,22,23]$, and Korea [24-26], two each in Germany $[27,28]$ and Italy $[29,30]$, one each in Taiwan [31], Spain [32], Netherlands [33], Romania [34], and France [35], and one combined study in USA and Italy [36]. Among them, two studies were prospective $[28,32]$ and others were retrospective. Five studies were performed on minimally invasive procedures (three robotic $[21,23,30]$ and two laparoscopic $[26,35])$. Indications for the central or distal pancreatectomy were benign pathologies like trauma and pancreatitis, benign and borderline tumors of pancreatic neck and proximal body, and few malignant cases (Table 2).

\section{Quantitative Data Synthesis}

Results of meta-analysis are included in Table 3.

4.1. Intraoperative Outcomes. Operation time was pooled from $20[7,14-19,21-29,31,34-36]$ studies and meta-analysis was done with the random effects model. CP was associated with significantly longer operation time (SMD: 1.03; 95\% CI 0.62 to 1.44; $P<0.001$ ) (Figure 2). CP was associated with significantly less blood loss when data were pooled from 18
[7, 14-19, 21, 22, 24-26, 28, 29, 31, 34-36] comparative studies with random effects model (SMD: $-0.34 ; 95 \%$ CI -0.58 to $-0.09 ; P=0.007$ ) (Figure 3). Transfusion requirement was pooled from $13[7,14,16-18,21,22,24,25,29,34-36]$ comparative studies, but the difference was not statistically significant (SMD: $0.69 ; 95 \%$ CI 0.47 to $1.01 ; P=0.059$ ) (Figure 4).

4.2. Postoperative Outcomes. Length of postoperative hospital stay (LOS) was compared in 23 [7, 14, 15, 17-34, 36, 37] studies and meta-analysis was done using a random effects model. CP had significantly longer postoperative LOS (SMD: 0.63 ; $95 \%$ CI 0.20 to $1.05 ; P<0.01$ ) (Figure 5 ).

Overall postoperative morbidity was recorded in 50.3\% (251/499) of patients following CP and 39.2\% (393/1002) of patients following DP. The most common complication was a postoperative pancreatic fistula. Reported data on overall complications in 21 [7, 14-18, 21-24, 26-36] studies showed that the complications were significantly higher after $\mathrm{CP}$ (RR: 1.30 ; $95 \%$ CI: 1.13 to $1.50 ; P<0.001$ ) than those after DP (Figure 6). Similarly, the overall pancreatic fistula was significantly higher after CP (RR: 1.41 ; 95\% CI: 1.20 to 1.66 ; $P<0.001$ ) [7, 14-36] (Figure 7). Overall pancreatic fistulas following CP and DP were 39.6\% (235/593) and 26.3\% (323/ 1226), respectively. Classification of the fistula was done according to the International Study Group for Pancreatic 


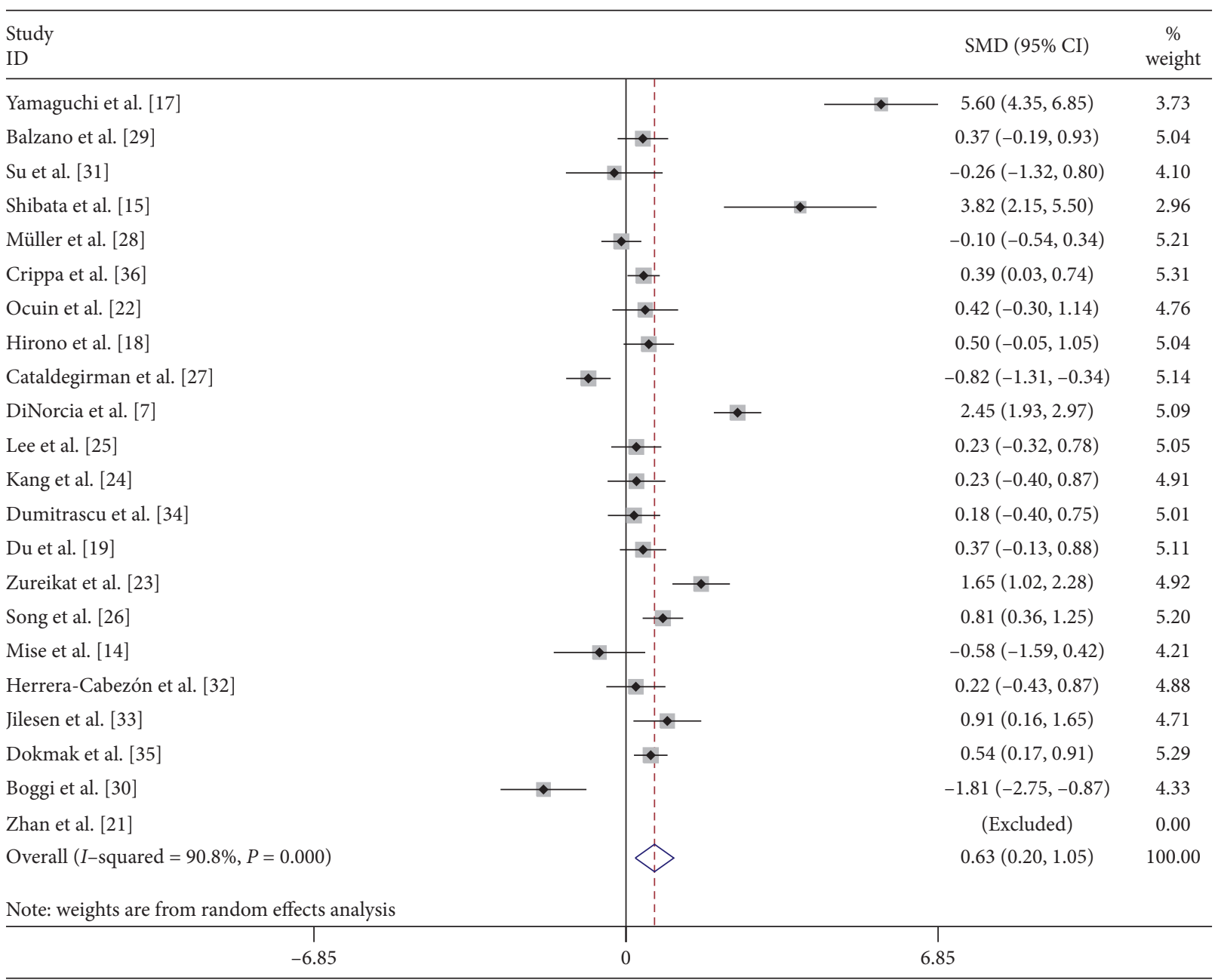

FIGURE 5: Forest plot comparing length of hospital stay (LOS) for central versus distal pancreatectomy.

Fistula (ISGPF), Clavien-Dindo classification, and amount of postoperative amylase drainage in some studies. Most of the pancreatic fistulas following CP or DP were successfully treated by percutaneous drainage and antibiotics.

Classification of the fistula was recorded in 12 $[7,18,20,22,23,26,30,32-36]$ studies with 345 and 825 patients undergoing CP and DP, respectively. Grade A fistulas following CP and DP were seen in 65 (18.8\%) and $81(9.8 \%)$ patients, respectively. Clinically relevant fistulas (Grade B +C) were seen in $23.1 \%(81 / 350)$ and $18.18 \%(150 / 825)$ patients with CP and DP, respectively. Pooled data from these studies showed that there was a significantly higher incidence of clinically relevant fistula after CP (RR: $1.64 ; 95 \%$ CI: 1.25 to $2.16 ; P<0.001$ ) (Figure 8). The data pooled from 11 studies indicated that $\mathrm{CP}$ had significantly higher risk of postoperative hemorrhage (RR: $1.90 ; 95 \%$ CI: 1.18 to $3.06 ; P<0.05$ ) (Figure 9).

Reoperation was compared in $15[7,14,16,21,23$, $24,27-30,32-36]$ studies, which showed that there was no significant difference in risk of reoperation between two groups (RR: 1.10 ; 95\% CI: 0.69 to 1.73 ) (Figure 10). Reoperation was performed in 23 patients who underwent
$\mathrm{CP}$ and 44 patients who underwent DP. Causes of reoperation after $\mathrm{CP}$ include postoperative hemorrhage $(n=10)$, anastomotic insufficiency $(n=2)$, splenic infarction $(n=1)$, tracheostomy for prolonged ventilation $(n=1)$, and unknown $(n=9)$. Causes of reoperation in DP were postoperative hemorrhage $(n=9)$, anastomotic insufficiency $(n=3)$, postoperative bowel obstruction $(n=2)$, intra-abdominal abscess $(n=2)$, splenic infarction $(n=1)$, ischemic cecal perforation $(n=1)$, wound dehiscence $(n=1)$, and unknown $(n=25)$. In unknown causes of reoperation, causes were not mentioned clearly. Mortality data were compared in 18 studies. Two cases of 30-day mortality were seen after $\mathrm{CP}$, one with postoperative hemorrhage and the other secondary to pulmonary failure. Only one case of 30-day mortality secondary to myocardial infarction was reported after DP. Comparative data of tumor recurrence was mentioned in 10 studies. Nine cases of recurrence were identified after CP and 21 cases after DP.

Data on perioperative mortality (30 days postoperative) was extracted from $3[22,28,32]$ studies. There were two mortalities in the CP group and one in the DP group. The cause of death after $\mathrm{CP}$ was postoperative hemorrhage and 


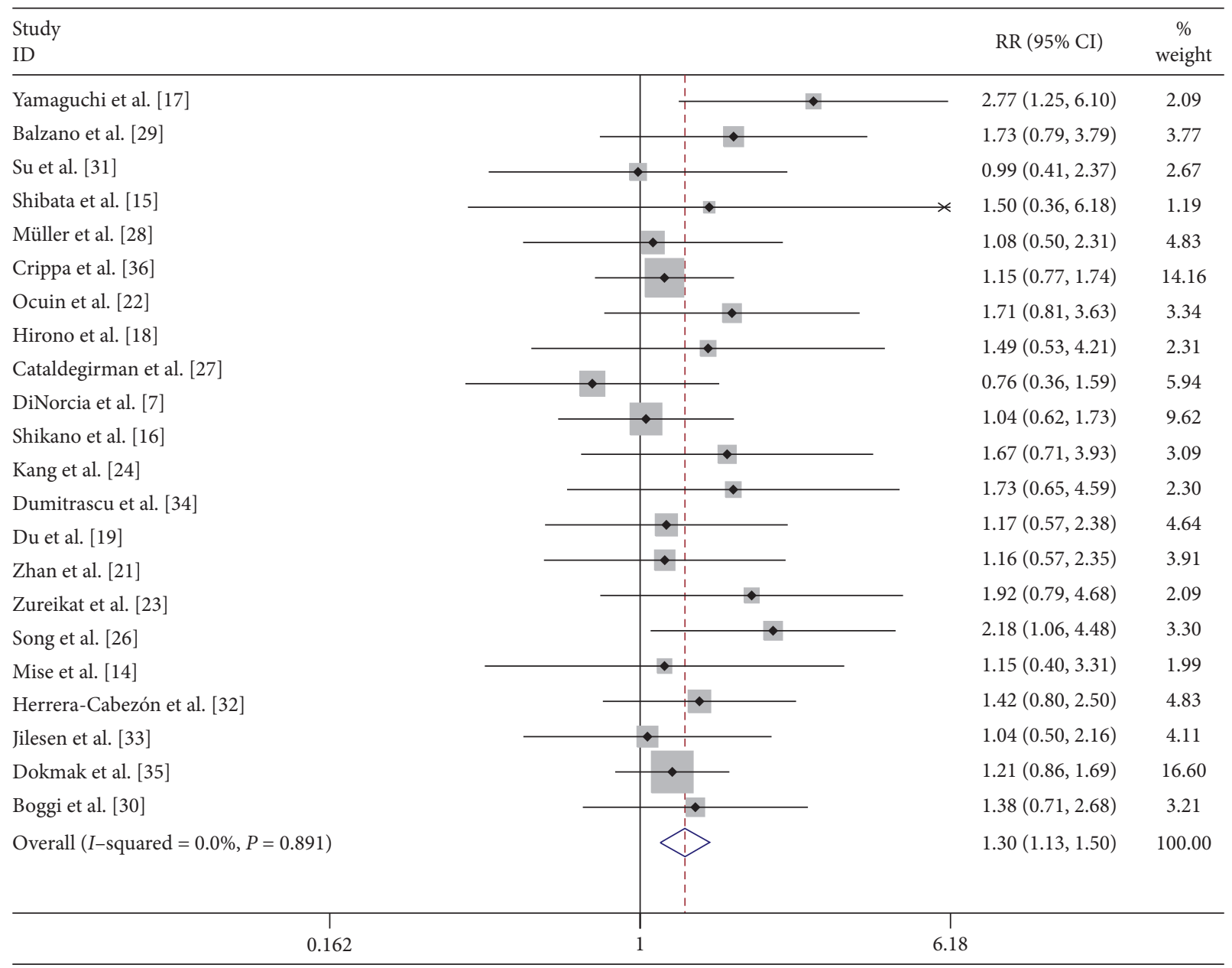

FIGURE 6: Forest plot comparing overall postoperative morbidity for central versus distal pancreatectomy.

pulmonary failure. The patient in the DP group died due to myocardial infarction. Pooled data showed that mortality in the two groups had no significant difference (RR: 3.31; 95\% CI: 0.52 to $21.32 ; P=0.207$ ) (Supplementary Figure 1 ).

4.3. Long-Term Outcomes. The postoperative endocrine function was assessed with the assessment of antidiabetic treatment, fasting blood glucose, hemoglobin A1c levels, oral glucose tolerance test, and World Health Organization criteria. All patients with preoperative diabetes were removed from the analysis. Overall incidence of postoperative new-onset diabetes was recorded in 18 [7, 15, 16, 18-20, 22-29, 31, 33, 34, 36] studies and was noted in 4.8\% (23/483) CP patients and 22.05\% $(153 / 694)$ DP patients. The pooled data of these studies showed that the risk of postoperative endocrine function impairment was significantly lower after CP (RR: $0.27 ; 95 \%$ CI: 0.18 to 0.40 ; $P<0.001$ ) (Figure 11). Data of insulin-dependent diabetes mellitus (IDDM) was compared in $6[7,16,18,19,22,36]$ studies and the pooled data demonstrated that patients in $\mathrm{CP}$ group were less likely to suffer from IDDM (RR: 0.15; 95\% CI: 0.06 to $0.42 ; P<0.001$ ) (Supplementary Figure 2).

The postoperative exocrine function was assessed by laboratory tests ( $\mathrm{p}$-aminobenzoic test, fecal chymotrypsin level, and pancreolauryl test), symptoms (weight loss, diarrhea, and steatorrhoea), and the need for pancreatic enzyme supplementation. Postoperative exocrine insufficiency was recorded in $9[16,18,19,22,27,28,33,34,36]$ studies with $8.9 \%$ (27/304) and $17.3 \%$ (56/324) following CP and DP, respectively. Pooled data of these studies indicated that patients in the CP group were less likely to suffer from exocrine insufficiency (RR: 0.56 ; $95 \%$ CI: 0.37 to 0.84 ; $P<0.05)$ (Figure 12).

Comparative data of tumor recurrence was pooled from $7[16,21,25,27,28,33,34]$ studies suggesting no significant difference between the two groups (RR: 1.02; 95\% CI: 0.48 to $2.20 ; P>0.05$ ) (Supplementary Figure 3 ).

\section{Heterogeneity and Publication Bias}

Significant heterogeneity (high $I$ square value $>30 \%$ and $P$ value $<0.05)$ was observed in three continuous variables (operation time, estimated blood loss, and length of hospital stay). All three outcomes were pooled on the random-effects model. Therefore, a sensitivity analysis was done by omitting 1 study at a time and the pooled RR was calculated for the remaining studies to identify the potential source of heterogeneity between studies, but no single study significantly 


\begin{tabular}{|c|c|c|c|}
\hline \multicolumn{2}{|l|}{$\begin{array}{l}\text { Study } \\
\text { ID }\end{array}$} & $\mathrm{RR}(95 \% \mathrm{CI})$ & \multirow{2}{*}{$\begin{array}{c}\% \\
\text { Weight } \\
0.98\end{array}$} \\
\hline Yamaguchi et al. [17] & 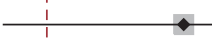 & $3.64(1.04,12.76)$ & \\
\hline Balzano et al. [29] & : & $2.67(0.86,8.27)$ & 2.26 \\
\hline Su et al. [31] & 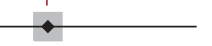 & $1.42(0.51,3.99)$ & 2.04 \\
\hline Shibata et al. [15] & 1 & $1.85(0.23,14.85)$ & 0.70 \\
\hline Müller et al. [28] & 1 & $0.77(0.18,3.23)$ & 2.24 \\
\hline Crippa et al. [36] & $\rightarrow$ & $1.36(0.80,2.34)$ & 10.49 \\
\hline Ocuin et al. [22] & $\frac{1}{1}$ & $3.81(0.92,15.81)$ & 1.16 \\
\hline Hirono et al. [18] & - & $1.46(0.75,2.84)$ & 5.73 \\
\hline Cataldegirman et al. [27] & & $0.85(0.28,2.58)$ & 3.35 \\
\hline DiNorcia et al. [7] & $\leftrightarrow \quad$ & $1.16(0.54,2.48)$ & 5.75 \\
\hline Lee et al. [25] & $\rightarrow$ & $1.38(0.46,4.15)$ & 2.23 \\
\hline Shikano et al. [16] & $\rightarrow$ & $1.88(0.68,5.22)$ & 2.60 \\
\hline Kang et al. [24] & $\longrightarrow$ & $2.17(0.61,7.70)$ & 1.63 \\
\hline Dumitrascu et al. [34] & 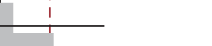 & $0.93(0.42,2.06)$ & 5.22 \\
\hline Xiang et al. [20] & 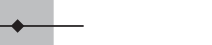 & $1.13(0.71,1.78)$ & 13.00 \\
\hline Du et al. [19] & $\rightarrow$ & $1.25(0.60,2.62)$ & 5.43 \\
\hline Zhan et al. [21] & & $1.14(0.53,2.48)$ & 4.12 \\
\hline Zureikat et al. [23] & & $1.59(0.97,2.59)$ & 7.07 \\
\hline Song et al. [26] & $\rightarrow$ & $2.10(0.74,5.95)$ & 2.08 \\
\hline Mise et al. [14] & & $1.80(0.19,16.66)$ & 0.60 \\
\hline Herrera-Cabezón et al. [32] & $\rightarrow$ & $1.89(0.92,3.88)$ & 3.20 \\
\hline Jilesen et al. [33] & $\rightarrow$ & $3.08(0.93,10.18)$ & 0.97 \\
\hline Dokmak et al. [35] & - & $1.18(0.77,1.80)$ & 14.10 \\
\hline Boggi et al. [30] & $\rightarrow$ & $1.39(0.64,3.02)$ & 3.03 \\
\hline Overall $(I$-squared $=0.0 \%, P=0.927)$ & & $1.41(1.20,1.66)$ & 100.00 \\
\hline $\begin{array}{c}1 \\
0.06\end{array}$ & & & \\
\hline
\end{tabular}

FIGURE 7: Forest plot comparing overall pancreatic fistula for central versus distal pancreatectomy.

affected the primary outcome or heterogeneity. This may be due to a difference in surgical skills among surgeons and postoperative management strategy. Publication bias was considered to be present when the $P$ value was $<0.1$. Assessment of publication bias of dichotomous data using the funnel plot showed symmetrical distribution and the Egger's test did not show any statistical significance.

\section{Discussion}

This meta-analysis included 24 studies involving 1819 patients and assessed the intraoperative and postoperative outcomes after CP or DP. Long operation time in the CP is due to its distinct anatomical location and complex surgical procedure. But one [29] study reported a comparatively shorter duration of operation in the $\mathrm{CP}$ group. In our study, on comparing intraoperative outcomes between the two procedures, estimated blood loss was statistically higher during distal pancreatectomy. Thus, a relatively higher number of patients required transfusion after distal pancreatectomy. In $\mathrm{CP}$, the method of reconstruction of the distal stump was recorded in 17 studies with 359 patients.
Pancreaticojejunostomy (PJ) was performed in 225 patients and pancreaticogastrostomy in 112 patients. The remaining 22 cases were treated with duct occlusion. In DP, the proximal stump was ligated in most of the cases, but, in a few cases, anastomosis of a remnant of the pancreatic head with the Roux-en-Y limb was done. The rate of fistula following different reconstruction techniques was not compared in most of the studies.

The difference in the cumulative incidence of overall postoperative morbidity after CP and DP was $50.3 \%$ and $39.2 \%$, respectively. Huge variation was observed in morbidity after $\mathrm{CP}$ among included studies ranging from $25.7 \%$ [27] to $92 \%$. The overall incidence of the postoperative pancreatic fistula and the clinically relevant postoperative pancreatic fistula was significantly higher after CP. In CP there is the formation of two stumps, reconstruction with two ductal-enteric anastomoses, and therefore a high risk of a pancreatic leak from the anastomosis. A recent meta-analysis by Ricci et al. indicated that the risk of the clinically relevant pancreatic fistula is relatively higher after reconstruction with PJ [38]. This may be a cause of a high incidence of 


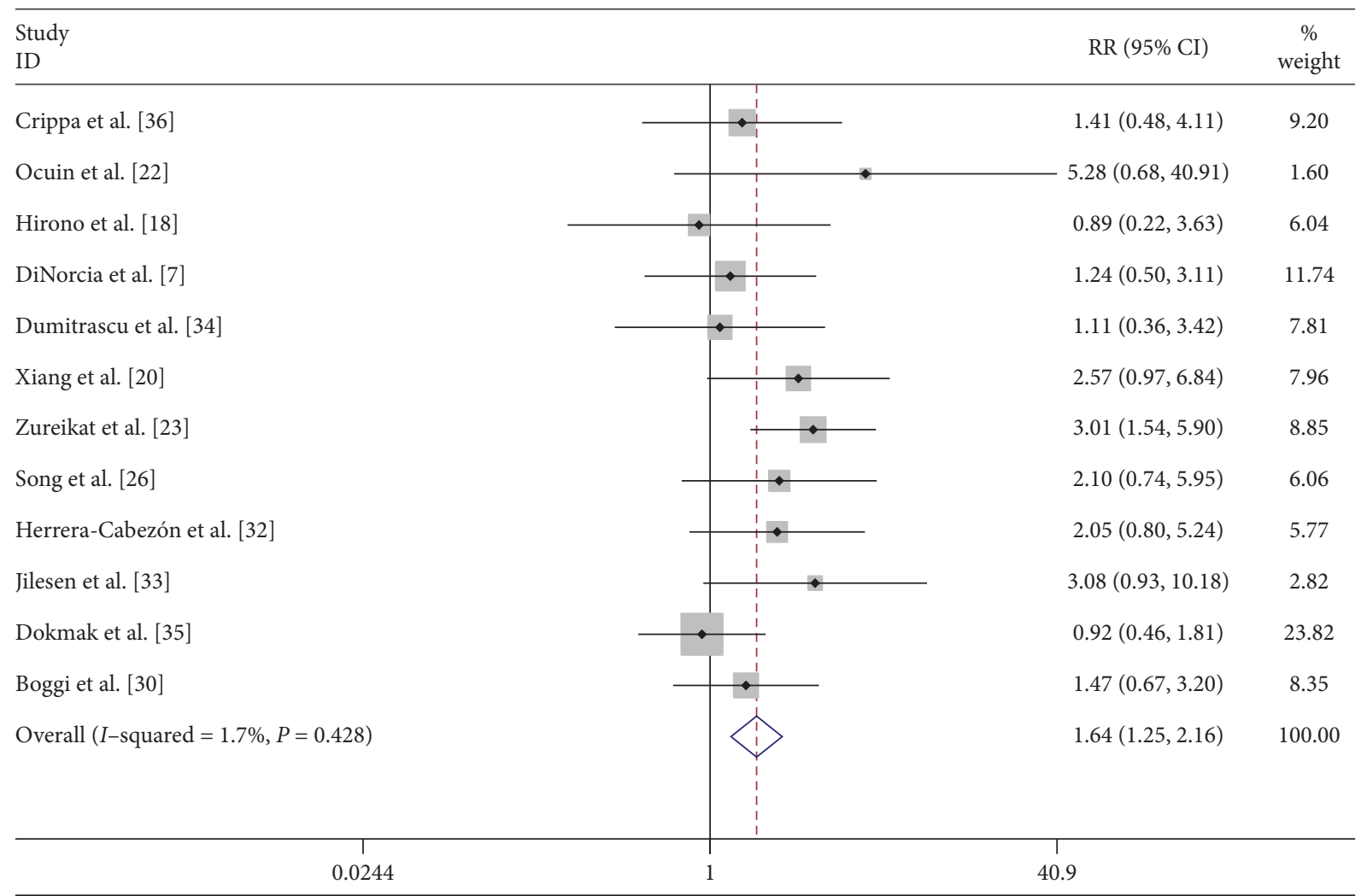

FIGURE 8: Forest plot comparing clinically relevant pancreatic fistula (Grade B $+C$ ) for central versus distal pancreatectomy.

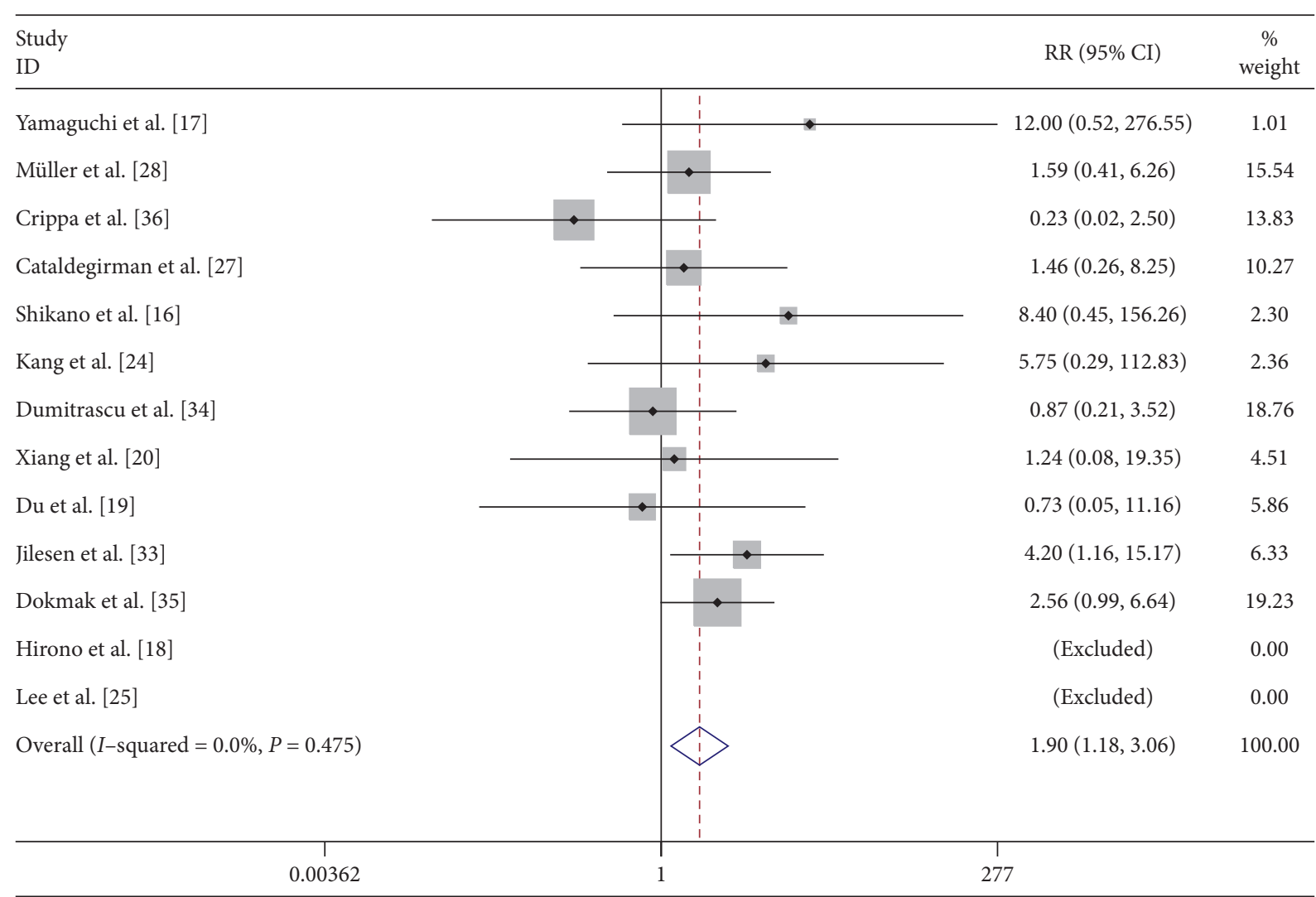

FIGURE 9: Forest plot comparing postoperative hemorrhage (POH) for central versus distal pancreatectomy. 


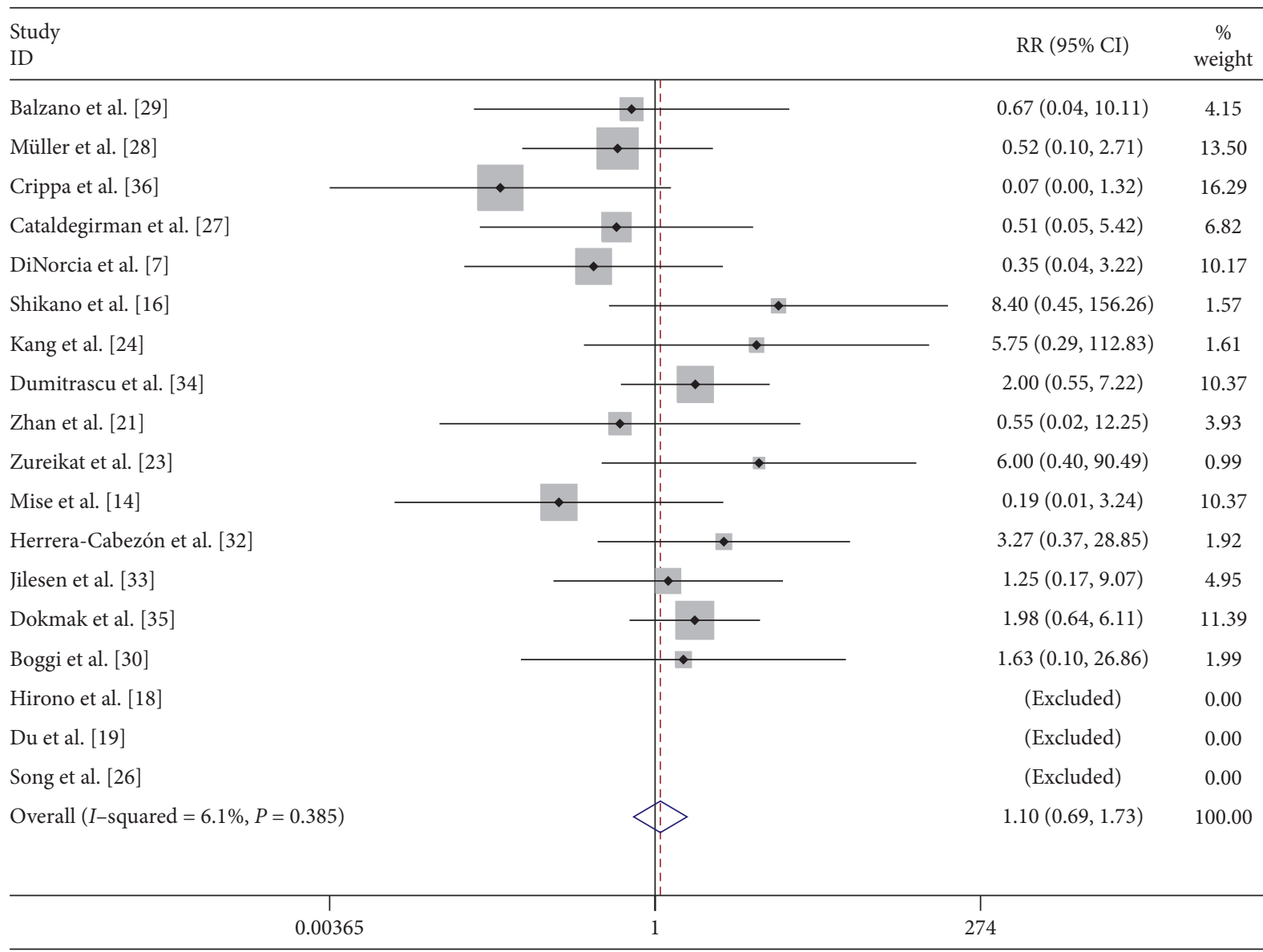

FIGURE 10: Forest plot comparing reoperation for central versus distal pancreatectomy.

postoperative pancreatic fistula after $\mathrm{CP}$ as $\mathrm{PJ}$ was performed in the majority of patients in our study. Clinically relevant fistula did not differ significantly between two groups in the previous meta-analysis (RR: 0.76 ; 95\% CI: $0.37-1.57 ; P>0.05$ ) [39], but our study showed significantly high incidence of clinically relevant pancreatic fistula (Grade B +C) (RR: 1.64; 95\% CI: 1.25 to 2.16; $P<0.001)$. For patient's safety, most of the intraoperative and postoperative outcomes favor DP over CP.

The incidence of postoperative hemorrhage was significantly high in the $\mathrm{CP}$ group. We believe segmental resection of a relatively large pancreatic neck tumor exposes an area of the portal vein (PV) near ducto-enteric anastomosis, hence, a pancreatic leak that may erode surrounding blood vessels and induce postoperative hemorrhage. In our study, 10 out of $23 \mathrm{CP}$ patients required reoperation for postoperative hemorrhage, but only 9 out of $44 \mathrm{DP}$ patients required reoperation for postoperative hemorrhage. Complex reconstruction techniques and exposed surrounding blood vessels might be the reason for reoperation in a relatively higher percentage of $\mathrm{CP}$ patients with $\mathrm{PO}$ hemorrhage. However, there was no significant difference in the overall rate of reoperation between the two groups. 30-day postoperative mortality was also higher in the $\mathrm{CP}$ group which was not statistically significant.
A significant difference in the incidence of postoperative new-onset endocrine insufficiency was seen between CP and DP ( $4.8 \%$ versus $22.05 \%$ ). Postoperative endocrine insufficiency was not observed in 9 studies $[15,17,20,24,25,28,31,34]$ after CP, but it was observed in all studies after DP. A recent nationwide database study on glucose metabolism after DP has shown 22.1\% incidence of new-onset diabetes. [40] Female gender, higher $\mathrm{BMI}$, and resection of pancreatic volume $>25 \%$ are the risk factors for postoperative endocrine impairment [8]. Incidence of postoperative endocrine insufficiency following DP was 19.5\% in studies performed in the Asian population [15-20, 24-26, 31] and $26.5 \%$ in studies performed in the western population $[7,22,27-29,33,34,36]$. The difference incidence of new-onset IDDM was even greater, for which the cumulative incidence was $0.9 \% \quad(2 / 227)$ after CP and $12.9 \% \quad(21 / 163)$ after DP $[7,16,18,19,22,36]$. The previous meta-analysis showed no significant difference in the incidence of postoperative exocrine failure (pooled RR: 0.59; 95\% CI: 0.32 to $1.07 ; P=0.082$ ) [41]. In contrast, our study showed a significant difference between the two groups (RR: 0.56 ; $95 \%$ CI: 0.37 to $0.84 ; P<0.05$ ). The incidence of postoperative exocrine failure after $\mathrm{CP}$ or $\mathrm{CP}$ varies widely, as it depends on preexisting pancreatic abnormality, the extent of resection, and presence of chronic pancreatitis $[28,42,43]$. Therefore, in selected patients who need preservation of the pancreatic parenchyma, $\mathrm{CP}$ is of utmost importance. 


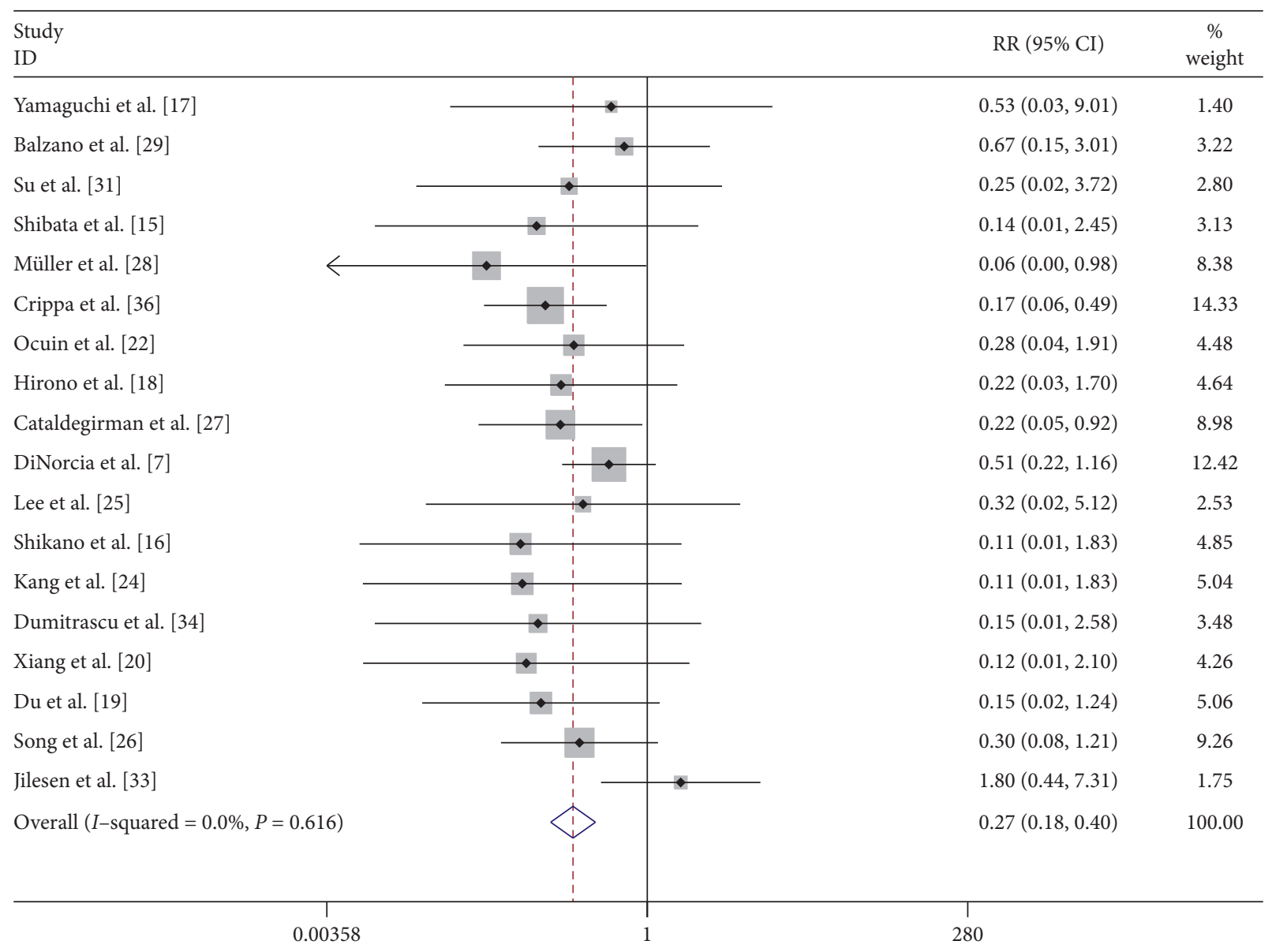

FIGURE 11: Forest plot comparing postoperative endocrine insufficiency for central versus distal pancreatectomy.

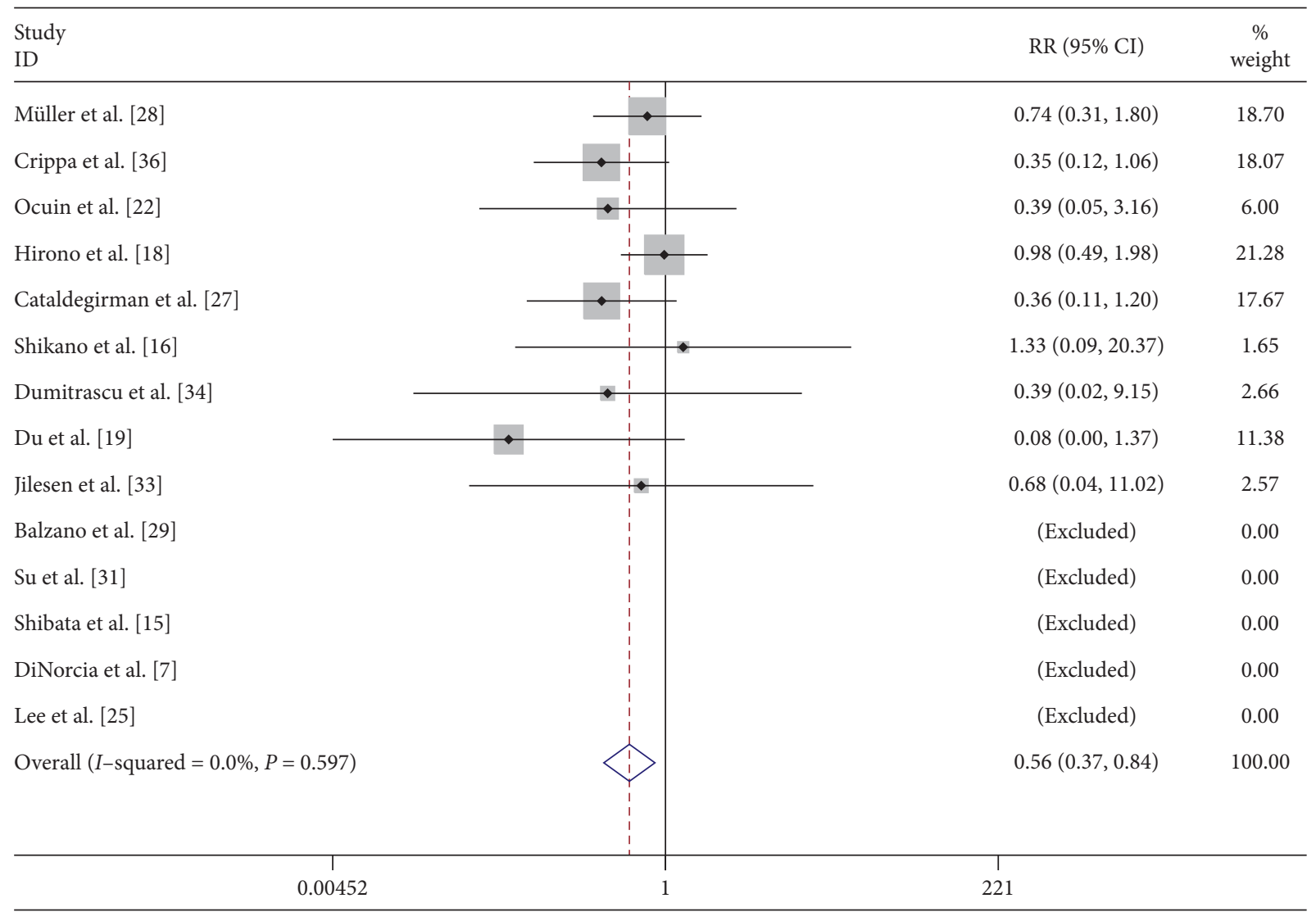


Tumor recurrence was observed in $1.52 \%$ of patients following $\mathrm{CP}$, among whom the majority of cases were IPMN. A study has shown that mucinous neoplasms have a $62.2 \%$ positive predictive value for malignancy, so intraoperative frozen-section analysis is necessary to obtain R0 resection and reduce the incidence of tumor recurrence $[44,45]$.

\section{Conclusion}

Our study suggests that pancreatic neck resection requires longer operation time, high rate of postoperative pancreatic fistula, and high morbidity and mortality, but less amount of normal parenchyma is resected in contrast to DP. The incidence of postoperative endocrine and exocrine insufficiency is relatively less after CP. Few previously published meta-analyses have shown that $\mathrm{CP}$ can be feasible for benign and low-malignant lesions of the pancreatic neck and proximal body. In contrast to those studies, our study showed that the incidence of serious postoperative morbidity (i.e., clinically relevant pancreatic fistula) was significantly high after CP. In our study, the cumulative incidence of postoperative endocrine insufficiency was relatively lower in Asians compared to the western population (19.5\% versus $26 \%$ ). We believe that postoperative diabetes can be well controlled with oral hypoglycemic drugs and insulin. However, regarding higher morbidity and mortality after $\mathrm{CP}$, it is still questionable for patient safety.

Although CP has the advantage of postoperative pancreatic function preservation, due to lengthy operation time, high rate of complications, and higher incidence of postoperative fistula. We conclude DP is a comparatively safe procedure compared to CP. Therefore, for tumors in pancreatic body and tail, DP is the safest, most feasible, and accepted procedure unless pancreatic parenchyma preservation is of utmost importance.

\section{Data Availability}

The data types used to support the findings of this study are included in the article and supplementary information files.

\section{Additional Points}

Significant heterogeneity existed in three continuous variables (operation time, blood loss, and length of hospital stay), which indicates the difference is surgical skills between surgeons, studies conducted in different countries, different sample sizes, and so on. This study lacks data for postoperative gastroparesis and only a few studies recorded data on tumor recurrence. Moreover, only prospective observational and retrospective studies were identified during the literature search, so this study may not be as reliable as metaanalysis was performed on clinical trials and international multicenter studies.

\section{Conflicts of Interest}

The authors have no conflicts of interest to declare.

\section{Authors' Contributions}

Parbatraj Regmi and Qing Yang and Hai-Jie Hu contributed equally to the study. Fu-Yu Li contributed to the study design and revision of the manuscript. All authors read and approved the final manuscript.

\section{Acknowledgments}

This study was funded by the Fundamental Research Funds for the Central Universities (2019SCUH), Science and Technology Support Project of Sichuan Province (2018JY0019), and Post-Doctor Research Project, West China Hospital, Sichuan University (2019HXBH025).

\section{Supplementary Materials}

Bias figure " " funnel plot: funnel plot for publication bias "data mean": collected data for meta-analysis. Supplementary Figure 1: forest plot comparing perioperative mortality for central versus distal pancreatectomy. Supplementary Figure 2: forest plot comparing postoperative insulin-dependent diabetes mellitus (IDDM) for central versus distal pancreatectomy. Supplementary Figure 3: forest plot comparing tumor recurrence for central versus distal pancreatectomy. (Supplementary Materials)

\section{References}

[1] S. F. Sener, A. Fremgen, H. R. Menck, and D. P. Winchester, "Pancreatic cancer: a report of treatment and survival trends for 100,313 patients diagnosed from 1985-1995, using the National Cancer Database 11 no competing interests declared," Journal of the American College of Surgeons, vol. 189, no. 1, pp. 1-7, 1999.

[2] M. Wagner, N. Dikopoulos, C. Kulli, H. Friess, and M. W. Buchler, "Standard surgical treatment in pancreatic cancer," Annals of Oncology: Official Journal of the European Society for Medical Oncology, vol. 10, no. 4, pp. 247-251, 1999.

[3] A. L. Warshaw and C. F.-d. Castillo, "Pancreatic carcinoma," New England Journal of Medicine, vol. 326, no. 7, pp. 455-465, 1992.

[4] C. Iacono, A. Ruzzenente, L. Bortolasi, and A. Guglielmi, "Central pancreatectomy: the dagradi serio lacono operation. Evolution of a surgical technique from the pioneers to the robotic approach," World Journal of Gastroenterology, vol. 20, no. 42, pp. 15674-15681, 2014.

[5] F. Motoi, S. Egawa, and M. Unno, "Middle pancreatectomy," Journal of Hepato-Biliary-Pancreatic Sciences, vol. 19, no. 2, pp. 148-151, 2012.

[6] R. A. Burkhart, S. M. Gerber, R. M. Tholey et al., "Incidence and severity of pancreatogenic diabetes after pancreatic resection," Journal of Gastrointestinal Surgery, vol. 19, no. 2, pp. 217-225, 2015.

[7] J. DiNorcia, L. Ahmed, M. K. Lee et al., "Better preservation of endocrine function after central versus distal pancreatectomy for mid-gland lesions," Surgery, vol. 148, no. 6, pp. 1247-1256, 2010.

[8] J. S. Kang, J.-Y. Jang, M. J. Kang et al., "Endocrine function impairment after distal pancreatectomy: incidence and related factors," World Journal of Surgery, vol. 40, no. 2, pp. 440-446, 2016. 
[9] H. A. Reber, "Middle pancreatectomy: why I rarely do it," Journal of Gastrointestinal Surgery, vol. 11, no. 6, pp. 730-732, 2007.

[10] M. Santangelo, A. Esposito, V. Tammaro et al., "What indication, morbidity and mortality for central pancreatectomy in oncological surgery? a systematic review," International Journal of Surgery, vol. 28, no. 1, pp. S172-S176, 2016.

[11] L. Shamseer, D. Moher, M. Clarke et al., "Preferred reporting items for systematic review and meta-analysis protocols (PRISMA-P) 2015: elaboration and explanation," BMJ, vol. 349, p. g7647, 2015.

[12] N. Luo, G. Liu, M. Li, H. Guan, X. Jin, and K. RandHendriksen, "Estimating an EQ-5D-5L value set for China," Value in Health, vol. 20, no. 4, pp. 662-669, 2017.

[13] X. Wan, W. Wang, J. Liu, and T. Tong, "Estimating the sample mean and standard deviation from the sample size, median, range and/or interquartile range," BMC Medical Research Methodology, vol. 14, p. 135, 2014.

[14] Y. Mise, N. Tanaka, T. Furuya et al., "The feasibility of central pancreatectomy for blunt pancreatic trauma: a comparative study of central and distal pancreatectomy," Hepato-Gastroenterology, vol. 61, no. 132, pp. 1107-1112, 2014.

[15] S. Shibata, T. Sato, H. Andoh et al., "Outcomes and indications of segmental pancreatectomy," Digestive Surgery, vol. 21, no. 1, pp. 48-53, 2004.

[16] T. Shikano, A. Nakao, Y. Kodera et al., "Middle pancreatectomy: safety and long-term results," Surgery, vol. 147, no. 1, pp. 21-29, 2010.

[17] K. Yamaguchi, K. Yokohata, M. Ohkido et al., "Which is less invasive-distal pancreatectomy or segmental resection?" International Surgery, vol. 85, no. 4, pp. 297-302, 2000.

[18] S. Hirono, M. Tani, M. Kawai et al., "A central pancreatectomy for benign or low-grade malignant neoplasms," Journal of Gastrointestinal Surgery, vol. 13, no. 9, pp. 1659-1665, 2009.

[19] Z.-Y. Du, S. Chen, B. S. Han, B. Y. Shen, Y. B. Liu, and C. H. Peng, "Middle segmental pancreatectomy: a safe and organ-preserving option for benign and low-grade malignant lesions," World Journal of Gastroenterology, vol. 19, no. 9, pp. 1458-1465, 2013.

[20] G. M. Xiang, C. L. Tan, H. Zhang, X. Ran, G. Mai, and X. B. Liu, "Central pancreatectomy for benign or borderline lesions of the pancreatic neck: a single centre experience and literature review," Hepato-Gastroenterology, vol. 59, no. 59, pp. 1286-1289, 2012.

[21] Q. Zhan, X.-X. Deng, B. Han et al., "Robotic-assisted pancreatic resection: a report of 47 cases," The International Journal of Medical Robotics and Computer Assisted Surgery, vol. 9, no. 1, pp. 44-51, 2013.

[22] L. M. Ocuin, J. M. Sarmiento, C. A. Staley et al., "Comparison of central and extended left pancreatectomy for lesions of the pancreatic neck," Annals of Surgical Oncology, vol. 15, no. 8, pp. 2096-2103, 2008.

[23] A. H. Zureikat, A. J. Moser, B. A. Boone, D. L. Bartlett, M. Zenati, and H. J. Zeh, "250 robotic pancreatic resections," Annals of Surgery, vol. 258, no. 4, pp. 554-562, 2013.

[24] C. M. Kang, J.-M. Lee, M. W. Kim, D. S. Yoon, J. S. Park, and W. J. Lee, "Experiences in central pancreatectomy," Digestive Surgery, vol. 28, no. 1, pp. 57-62, 2011.

[25] S. E. Lee, J.-Y. Jang, D. W. Hwang, K. U. Lee, and S.-W. Kim, "Clinical efficacy of organ-preserving pancreatectomy for benign or low-grade malignant potential lesion," Journal of Korean Medical Science, vol. 25, no. 1, pp. 97-103, 2010.

[26] K. B. Song, S. C. Kim, K.-M. Park et al., "Laparoscopic central pancreatectomy for benign or low-grade malignant lesions in the pancreatic neck and proximal body," Surgical Endoscopy, vol. 29, no. 4, pp. 937-946, 2015.

[27] G. Cataldegirmen, C. G. Schneider, D. Bogoevski et al., "Extended central pancreatic resection as an alternative for extended left or extended right resection for appropriate pancreatic neoplasms," Surgery, vol. 147, no. 3, pp. 331-338, 2010.

[28] M. Müller, H. Friess, J. Kleeff et al., "Middle segmental pancreatic resection," Annals of Surgery, vol. 244, no. 6, pp. 909-920, 2006.

[29] G. Balzano, A. Zerbi, P. Veronesi, M. Cristallo, and V. Di Carlo, "Surgical treatment of benign and borderline neoplasms of the pancreatic body," Digestive Surgery, vol. 20, no. 6, pp. 506-510, 2003.

[30] U. Boggi, N. Napoli, F. Costa et al., "Robotic-assisted pancreatic resections," World Journal of Surgery, vol. 40, no. 10, pp. 2497-2506, 2016.

[31] C. H. Su, Y. M. Shyr, W. Y. Lui, and F. K. P'Eng, "Surgical treatment for serous cystadenoma of pancreas-segmental pancreatectomy or conventional resection?" Hepato-Gastro enterology, vol. 51, no. 56, pp. 595-598, 2004.

[32] F. J. Herrera-Cabezon, P. Sanchez-Acedo, C. Zazpe-Ripa, A. Tarifa-Castilla, and J. M. Lera-Tricas, "Quality standards in 480 pancreatic resections: a prospective observational study," Revista Espanola de Enfermedades Digestivas: Organo Oficial de la Sociedad Espanola de Patologia Digestiva, vol. 107, no. 3, pp. 143-151, 2015.

[33] A. P. J. Jilesen, C. H. J. van Eijck, O. R. C. Busch, T. M. van Gulik, D. J. Gouma, and E. J. M. N. van Dijkum, "Postoperative outcomes of enucleation and standard resections in patients with a pancreatic neuroendocrine tumor," World Journal of Surgery, vol. 40, no. 3, pp. 715-728, 2016.

[34] T. Dumitrascu, A. Scarlat, M. Ionescu, and I. Popescu, "Central pancreatectomy versus spleen-preserving distal pancreatectomy: a comparative analysis of early and late postoperative outcomes," Digestive Surgery, vol. 29, no. 5, pp. 400-407, 2012.

[35] S. Dokmak, B. Aussilhou, F. S. Ftériche, J. Belghiti, and A. Sauvanet, "Hanging maneuver for stomach traction in laparoscopic distal pancreatic resections: an original technique applied in 218 patients," Digestive Surgery, vol. 34, no. 2 , pp. 89-94, 2017.

[36] S. Crippa, C. Bassi, A. L. Warshaw et al., "Middle pancreatectomy," Annals of Surgery, vol. 246, no. 1, pp. 69-76, 2007.

[37] W. Pratt, S. Maithel, T. Vanounou, M. Callery, and C. Vollmer Jr., "Postoperative pancreatic fistulas are not equivalent after proximal, distal, and central pancreatectomy," Journal of Gastrointestinal Surgery, vol. 10, no. 9, pp. 1264-1279, 2006.

[38] C. Ricci, R. Casadei, G. Taffurelli, C. A. Pacilio, D. Beltrami, and F. Minni, "Is pancreaticogastrostomy safer than pancreaticojejunostomy after pancreaticoduodenectomy? a metaregression analysis of randomized clinical trials," Pancreatology, vol. 17, no. 5, pp. 805-813, 2017.

[39] S. B. Xu, Y. P. Zhu, W. Zhou, K. Xie, and Y. P. Mou, "Patients get more long-term benefit from central pancreatectomy than distal resection: a meta-analysis," European Journal of Surgical Oncology (EJSO), vol. 39, no. 6, pp. 567-574, 2013.

[40] J. M. Wu, T. W. Ho, C. Y. Yang, P. H. Lee, and Y. W. Tien, "Changes in glucose metabolism after distal pancreatectomy: a nationwide database study," Oncotarget, vol. 9, no. 13, pp. 11100-11108, 2018.

[41] C. Iacono, G. Verlato, A. Ruzzenente et al., "Systematic review of central pancreatectomy and meta-analysis of centralversusdistal 
pancreatectomy," British Journal of Surgery, vol. 100, no. 7, pp. 873-885, 2013.

[42] S. Ikeda, S. Matsumoto, K. Maeshiro, R. Miyazaki, K. Okamoto, and Y. Yasunami, "Segmental pancreatectomy for the diagnosis and treatment of small lesions in the neck or body of the pancreas," Hepato-Gastroenterology, vol. 42, no. 5, pp. 730-733, 1995.

[43] N. Rotman, B. Sastre, and P. L. Fagniez, "Medial pancreatectomy for tumors of the neck of the pancreas," Surgery, vol. 113 , no. 5, pp. 532-535, 1993.

[44] G. Marchegiani, S. Andrianello, A. Borin et al., "Systematic review, meta-analysis, and a high-volume center experience supporting the new role of mural nodules proposed by the updated 2017 international guidelines on IPMN of the pancreas," Surgery, vol. 163, no. 6, pp. 1272-1279, 2018.

[45] A. Sauvanet, C. Partensky, B. Sastre et al., "Medial pancreatectomy: a multi-institutional retrospective study of 53 patients by the french pancreas club," Surgery, vol. 132, no. 5, pp. 836-843, 2002. 